\title{
The Effects of Wheel-Running Using the Upper Limbs Following Immobilization After Inducing Arthritis in the Knees of Rats
}

\author{
Ying TONG ${ }^{1}$, Kumiko ISHIKAWA ${ }^{2}$, Ryo SASAKI ${ }^{3,4}$, Izumi TAKESHITA ${ }^{1}$, Junya \\ SAKAMOTO $^{1}$, Minoru OKITA ${ }^{1,3}$
}

${ }^{1}$ Department of Physical Therapy Science, Nagasaki University Graduate School of Biomedical Sciences, Nagasaki, Japan, ${ }^{2}$ Department of Rehabilitation, Nagasaki University Hospital, Nagasaki, Japan, ${ }^{3}$ Department of Locomotive Rehabilitation Science, Nagasaki University Graduate School of Biomedical Sciences, Nagasaki, Japan, ${ }^{4}$ Department of Rehabilitation, Juzenkai Hospital, Nagasaki, Japan

Received March 26, 2020

Accepted November 10, 2020

Epub Ahead of Print January 14, 2021

\section{Summary}

This study investigated the effects of wheel-running using the upper limbs following immobilization after inducing arthritis in the knees of rats. Forty male Wistar rats (aged 8 weeks) divided into four groups randomly: arthritis (AR), immobilization after arthritis (Im), wheel-running exercise with the upper limbs following immobilization after arthritis induction (Im+Ex) and sham arthritis induction (Con). The knee joints of the Im and Im+Ex groups were immobilized with a cast for 4 weeks. In the Im+Ex group, wheel-running exercise was administered for $60 \mathrm{~min} /$ day ( 5 times/week). The swelling and the pressure pain threshold (PPT) of the knee joint were evaluated for observing the condition of inflammatory symptoms in affected area, and the paw withdraw response (PWR) was evaluated for observing the condition of secondary hyperalgesia in distant area. Especially, in order to evaluate histological inflammation in the knee joint, the number of macrophage (CD68-positive cells) in the synovium was examined. The expression of calcitonin gene-related peptide (CGRP) in the spinal dorsal horn (L2-3 and L4-5) was examined to evaluate central sensitization. The Im+Ex group showed a significantly better recovery than the Im group in the swelling, PPTs, and PWRs. Additionally, CGRP expression of the spinal dorsal horn (L2-3 and L4-5) in the Im+Ex group was significantly decreased compared with the Im group. According to the results, upper limb exercise can decrease pain in the affected area, reduce hyperalgesia in distant areas, and suppress the central sensitization in the spinal dorsal horn by triggering exerciseinduced hypoalgesia $(\mathrm{EIH})$.

\section{Key words}

Arthritis - Wheel-running exercise - Immobilization • Hyperalgesia • Central sensitization

\section{Corresponding author}

M. Okita, Department of Physical Therapy Science, Nagasaki University Graduate School of Biomedical Sciences, 1-7-1 Sakamoto, Nagasaki, 852-8520 Japan. Fax: +81-95-8197965. E-mail: mokita@nagasaki-u.ac.jp

\section{Introduction}

Acute joint inflammation is one of the known causes of chronic pain (Neugebauer et al. 2007), and often occurs after damage to menisci and ligaments due to sports injuries, septic and surgical procedures. In the acute stages, the affected peripheral tissues become inflamed. This is in response to the tissue repair process. The inflammatory reactions include redness, local heat, pain, and a variety of other symptoms. Conversely, when noxious stimulation is continuously applied to the spinal cord due to inflammation of peripheral tissues, various changes occur in the dorsal horn of the spinal cord leading to central sensitization (Radhakrishnan et al. 2003). Central sensitization in the spinal cord is a phenomenon of excessive excitement (hyperexcitability), and can be a cause of chronic pain. In rehabilitative strategies for acute arthritis, therefore, it is important to reduce musculoskeletal pain caused by inflammation and prevent 
the occurrence of central sensitization. However, if the inflammation of the affected area is remarkable, immobilization is performed for a certain period. In these instances, approaching the affected area is often difficult. Pervious study reported that excessive immobilization after arthritis can lead to significant pain in affected and distant areas, and central sensitization (Nakabayashi et al. 2016). Therefore, it is important to consider rehabilitative strategies, such as therapeutic exercise, for sites outside the affected areas.

Multiple clinical studies have proven that exercise can decrease pain symptoms and improve the body functions of patients with chronic pain (Tajerian and Clark 2017). For instance, regular walking has been recommended as an effective form of exercise or activity to relieve chronic musculoskeletal pain (O'Connor et al. 2015). This phenomenon is known as exercise-induced hypoalgesia (EIH) (Kami et al. 2017). In addition, EIH has been shown to be induced by both painful and non-painful movements (Vaegter et al. 2014). Pervious study showed that the effect of EIH can even cause systemic analgesic effects (Naugle et al. 2012). Interestingly, recent studies have shown that voluntary exercises, such as wheel-running, were much more analgesic than forced exercises during treadmill running (Kami et al. 2015).

Based on the information gleaned from these previous studies, the research presented herein used a rat experimental model of acute arthritis to simulate a clinical setting that required immobilization due to marked acute inflammation of an affected area. The effects of wheel-running using the upper limbs following immobilization after inducing acute inflammation in the knees of rats were also examined. We hypothesized that upper limb exercises would increase the pain threshold and decrease central sensitization due to immobilization after inducing joint inflammation.

\section{Methods}

\section{Animals}

The animals used in this study were male Wistar rats ( $\mathrm{n}=40$; 8 weeks old) provided by CLEA Japan, Inc., (Tokyo, Japan) and were randomly divided into four groups: (1) arthritis (AR, $n=10)$; (2) immobilization after arthritis (Im, n=10); (3) wheel-running exercise with the upper limbs following immobilization after arthritis induction ( $\mathrm{Im}+\mathrm{Ex}, \mathrm{n}=10)$; and (4) sham arthritis induction (Con, $\mathrm{n}=10$ ). All rats were housed in plastic cages and maintained on a $12 \mathrm{~h}$ light/dark cycle. Food and water were available ad libitum. All treatments were administered using an anesthetic agent $(1.875 \mathrm{ml}$ medetomidine hydrochloride (Kyoritu Pharma Co., Ltd., Tokyo, Japan) mixed with $2.0 \mathrm{ml}$ midazolam (Sandoz Pharma Co., Ltd., Tokyo, Japan) and $2.5 \mathrm{ml}$ butorphanol (Meiji Seika Pharma Co., Ltd., Tokyo, Japan) adjusted to a volume of $18.625 \mathrm{ml}$ with sterilized water (Kawai et al. 2011). The prepared anesthetic agent was administered intraperitoneally to the rats at a volume of $0.05 \mathrm{ml} / \mathrm{g}$ body weight.

All procedures were approved by the Nagasaki University Animal Care Committee. The Ethics Review Committee for Animal Experimentation of Nagasaki University approved all experiments (approval number: 1803291442-2).

\section{Arthritis induction}

Animal model in this study simulated a clinical setting that requires immobilization due to marked acute inflammation of an affected area. Therefore, the carrageenan model, which is commonly used for experimental acute arthritis, was adopted in this study (Radhakrishnan et al. 2003, Nakabayashi et al. 2016, Ishikawa et al. 2019).

Rats in the AR, Im, and Im+Ex groups were anesthetized and subsequently received a single injection of a $300 \mu \mathrm{l}$ mixture of $3 \%$ kaolin and $3 \%$ carrageenan (Sigma Chemical Co., St. Louis, MO, USA) anteriorly in the right knee joint cavity (Nakabayashi et al. 2016). The rats in the Con group received a sham injection of a $300 \mu 1$ saline.

\section{Immobilization}

In the Im and Im+Ex groups, the right leg in full extension of the knee joint and full plantar flexion of the ankle joint was immobilized using a plaster cast for 4 weeks post-injection. The left leg was not immobilized. The plaster casts were replaced at least every 2 or 3 days to prevent loosening and/or edema in their hind paws. The rats were able to move freely in the cage by using the three limbs that were not immobilized.

Application of wheel-running exercises using upper limbs Before the experiment, rats in the Im+Ex group were acclimated to the voluntary wheel-running exercise (10 $\mathrm{min} /$ day for 1 week). Exercise was initiated 1 day post-injection and a rat wheel-running measuring device was utilized (Natsume Seisakusho Co., Ltd., Tokyo, Japan). Rats in the Im+Ex group were individually placed in homemade restraints and then situated in the wheel- 
running measuring device. These rats were able to perform voluntary wheel-running exercises using their upper limbs. The exercises were performed for $60 \mathrm{~min} /$ day, 5 days/week, for 4 weeks.

During the 4-week experiments, the following tests were evaluated at baseline, and on the $1^{\text {st }}$ and $3^{\text {rd }}$ days, and the $1^{\text {st }}, 2^{\text {nd }}, 3^{\text {rd }}$, and $4^{\text {th }}$ week post-injection.

\section{Swelling of the knee joint}

To monitor joint swelling changes over time, the transverse diameter of the right knee joint was measured using a manual caliper. During the measurements, the knee joints were held in their maximum extended positions.

\section{The pressure pain threshold (PPT)}

The pressure pain threshold (PPT) of the inflamed knee joint was assessed using a Randall-Selitto apparatus (Ugo Basile, Varese, Italy). The rats were placed into a sock with their hind paws protruding and were restrained using the evaluator's hand. The rounded tip of the transducer probe (base diameter $=9 \mathrm{~mm}$ ) was applied to the lateral side of the knee joint with linearly increasing pressure (48 g/s). The threshold was defined as the force required to elicit the hind limb flection reflex or vocalization. Seven measurements were taken at intervals of at least $3 \mathrm{~min}$, and the average of 5 measurements (excluding the maximum and minimum) was recorded to attain the PPTs.

\section{The paw withdrawal response (PWR)}

Mechanical hyperalgesia of the hind paws was tested using von Frey filaments (VFF; North Coast Medical, Morgan Hill, CA, USA) after the animals were individually placed in a homemade restrainer (Nakano et al. 2012). This technique was employed because range of motion (ROM) limitations of the hip, knee, and ankle joint prevented the immobilized rats from placing their right hind paws on the ground. All rats were allowed to acclimate for $20 \mathrm{~min}$ prior to testing. The glabrous skin of the hind paw was probed 10 times using $4 \mathrm{~g}$ and $15 \mathrm{~g}$ VFFs at $10 \mathrm{~s}$ intervals. The lifting or pulling back of the paw or vocalization was considered a paw withdrawal response (PWR). The $4 \mathrm{~g}$ and $15 \mathrm{~g}$ VFFs were used to ascertain mechanical allodynia and hyperalgesia, respectively (Peleshok and Ribeiro-da-Silva 2011).

\section{Tissue sampling}

Four weeks post-injection, all rats were anesthetized and the right knee joint and the spinal cord
(L2-3, L4-5) of each rat was removed following transcardial perfusion with saline and $4 \%$ paraformaldehyde dissolved in $0.01 \mathrm{M}$ phosphate buffer (PB; $\mathrm{pH}$ 7.4). The right knee joint was decalcified with $10 \%$ ethylenediaminetetraacetic acid in $0.01 \mathrm{M}$ PB, pH 7.4. Each specimen was embedded in paraffin. Spinal cords were soaked for $24 \mathrm{~h}$ in $10 \%$ sucrose, followed by $24 \mathrm{~h}$ in $30 \%$ sucrose. The tissues were embedded in optimal cutting temperature compound, frozen, and stored at $-80{ }^{\circ} \mathrm{C}$.

Analysis of macrophages in the synovium of the right knee joint

Two sagittal sections ( $5 \mu \mathrm{m}$ thick) per rat were subjected to an antigen retrieval step by incubation in $0.01 \mathrm{M}$ citrate buffer, followed by incubation with $0.3 \%$ hydrogen peroxide $\left(\mathrm{H}_{2} \mathrm{O}_{2}\right)$ dissolved in methanol for $30 \mathrm{~min}$ at room temperature. The sections were blocked with $1 \%$ bovine serum albumin in phosphate buffered saline (PBS) for 60 min. Sections were then incubated with a mouse monoclonal anti-CD68 antibody (1:3000; AbD Serotec, Raleigh, NC, USA) overnight at room temperature, followed by incubation with biotinylated horse anti-mouse IgG (H+L) (1:3000; Vector Laboratories, BA-2000; Burlingame, CA, USA). Each section was stained using an avidin-biotin complex method (Vectastain Elite ABC kit; Vector Laboratories, Burlingame, CA, USA), and then visualized with a metalenhanced DAB substrate kit (Thermo Fisher Scientific Inc., Waltham, MA, USA). Each section was then stained with $1 \%$ methyl green. The anterior and posterior synovium sections were photographed at $400 \times$ magnification with a digital camera (DS-Ri1, Nikon Instruments Inc., Edgewood, NY, USA). For all images, the number of CD68-positive cells and areas were measured and the number of positive cells $/ \mathrm{mm}^{2}$ was calculated.

\section{Analysis of CGRP in the spinal dorsal horn}

To inhibit endogenous peroxidases, frozen spinal cord sections (10 $\mu \mathrm{m}$ thick) were incubated for $30 \mathrm{~min}$ at room temperature with $0.3 \% \mathrm{H}_{2} \mathrm{O}_{2}$ in methanol. Next, sections were blocked for 30 min with $5 \%$ bovine serum albumin in PBS, followed by incubation with an antiCGRP polyclonal antibody (1:3000; ImmunoStar Inc., Hudson, WI, USA) overnight at room temperature. The sections were then incubated with goat anti-rabbit IgG conjugated to Texas Red® (1:2000, Vector Laboratories, Burlingame, CA, USA) for $60 \mathrm{~min}$ at room temperature. Quantitative evaluation of CGRP expression in the ipsilateral dorsal horn was performed using an image- 
analysis software (NIS-Element ver. 3, Nikon Instruments Inc., Edgewood, NY, USA). Micrographs were obtained at $200 \times$ magnification and the spinal dorsal horn was divided into superficial (lamina I-II) and deep (lamina III-VI) layers according to previously described criteria (Molander et al. 1989). Total brightness in the images was calculated, and the data were divided by the area of each layer. The fluorescence intensities were evaluated using 5 sections per rat.

\section{Statistical analysis}

All data are presented as the mean \pm standard deviations (SD). Statistical analyses were performed using SPSS 22.0 statistical software. The level of significance was set at $P<0.05$. Differences among groups were assessed utilizing the Kruskal-Wallis Test or a one-way analysis of variance (ANOVA), followed by Steel-Dwass or Bonferroni post hoc test.

\section{Results}

\section{Changes in swelling}

Prior to the injections, the transverse diameter of the right knees was no significant differences among the 4 groups. On the $1^{\text {st }}$ day post-injection, AR, Im, and Im+Ex groups were significantly higher than Con group with sham injections. The swelling of the AR, Im, and Im+Ex groups began to recover after the $1^{\text {st }}$ week postinjection; however, AR group was significantly higher than Con group until the $4^{\text {th }}$ week. The swelling in the Im and Im+Ex groups returned to the same level as that in the Con group at the $3^{\text {rd }}$ week (Fig. 1a).
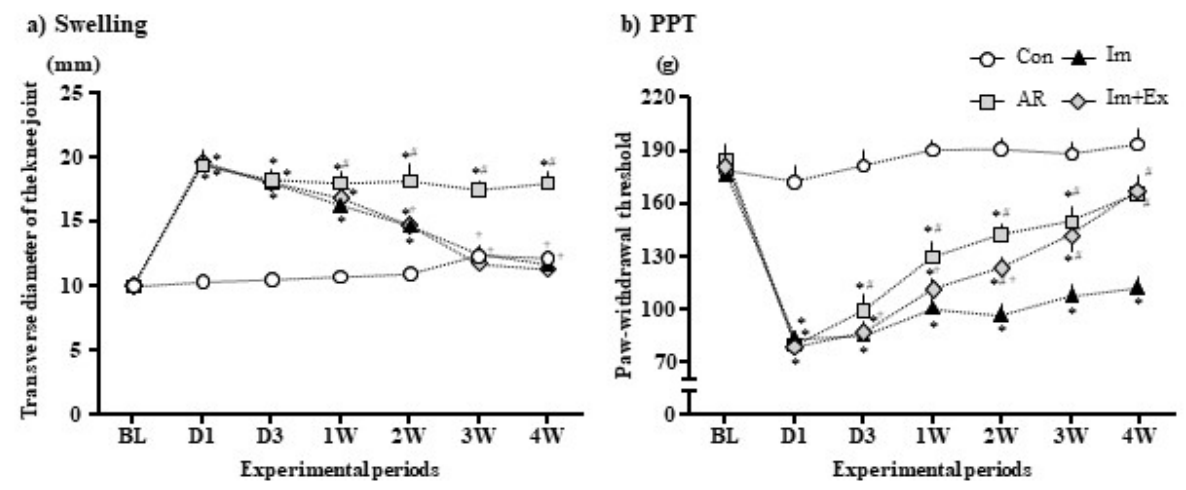

Fig. 1. Changes in swelling and PPT of the knee joint in all groups. (a) Changes in swelling. (b) Changes of PPT. Data are mean \pm SD. * significantly different from the Con group $(p<0.05) ;{ }^{+}$significantly different from the AR group $(p<0.05) ; \quad$ " significantly different from the Im group $(p<0.05)$. PPT: pressure pain threshold.

\section{Changes in PPT}

Prior to the injections, the PPT was no significant differences among the 4 groups. On the $1^{\text {st }}$ day post-injection, AR, Im, and Im+Ex groups were significantly lower than Con group. The PPTs in the AR, $\mathrm{Im}$, and $\mathrm{Im}+\mathrm{Ex}$ groups began to recover on the $3^{\text {rd }}$ day. In the $2^{\text {nd }}$ week, the PPT in the Im+Ex group had significantly recovered compared with that in the Im group. In the $3^{\text {rd }}$ week, the PPT in the $\mathrm{Im}+\mathrm{Ex}$ group gradually recovered to the same level as that of the AR group with no significant differences between the 2 groups. However, the PPT in the Im group was significantly lower than those in the other groups from the $1^{\text {st }}$ week to the $4^{\text {th }}$ week (Fig. $1 \mathrm{~b}$ ).

\section{Changes in PWR}

Prior to the injections, the PWRs using $4 \mathrm{~g}$ VFFs were no significant differences among the 4 groups. On the $1^{\text {st }}$ day post-injection, $A R, I m$, and $\mathrm{Im}+\mathrm{Ex}$ groups were significantly higher than Con group. The PWRs in the AR and Im+Ex groups began to recover at the $3^{\text {rd }}$ day or the $1^{\text {st }}$ week while the Im group began to recover at the $3^{\text {rd }}$ week. In the $2^{\text {nd }}$ week, Im group was significantly higher than those of the AR and Im+Ex groups. The PWRs in the Im group had not recovered at the $4^{\text {th }}$ week and the PWR was significantly higher than those in the AR, Im+Ex, and Con groups (Fig. 2a).

Prior to the injections, the PWRs using $15 \mathrm{~g}$ VFFs were no significant differences among the 4 groups. AR and Im+Ex groups began to recover from the $3^{\text {rd }}$ day while Im group began to recover at the $3^{\text {rd }}$ week. In the $1^{\text {st }}$ week, Im group was significantly higher than those of the AR and Im+Ex groups, and this tendency was consistent throughout the 4-week experimental period. The PWR in the Im group had not recovered at the $4^{\text {th }}$ week, and its PWR was significantly higher than those of the AR, Im+Ex, and Con groups (Fig. 2b).

\section{Expression of macrophages in the synovium of the knee}

In the AR, Im, and Im+Ex groups, large numbers of CD68-positive cells in the synovium were observed compared with the Con group (Fig. 3a). The numbers of 
CD68-positive cells in the synovium of the AR, Im, and Im+Ex groups were significantly increased compared with that of the Con group, with no significant differences among the 3 groups (Fig. 3b).

\section{Expression of CGRP in the spinal dorsal horn}

In the spinal dorsal horns at L2-3, CGRP-positive neural fibers were clearly observed in the deep layers in the AR, Im, and Im+Ex groups (Fig. 4a). In the superficial layer, there were no significant differences among the 4 groups. In the deep layer, the expression of CGRPs in the AR, Im, and Im+Ex groups significantly increased compared to that of the Con group. AR and Im+Ex groups significantly decreased when compared with the Im group, with no significant differences between the AR and Im+Ex groups (Fig. 4b). a) Von frey $4 g$

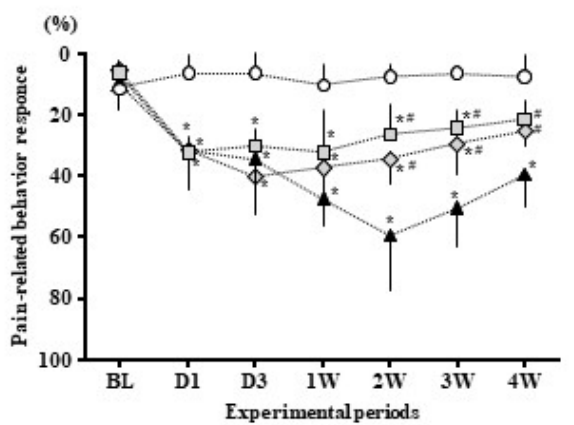

a) Immunohistochemical staining of macrophages

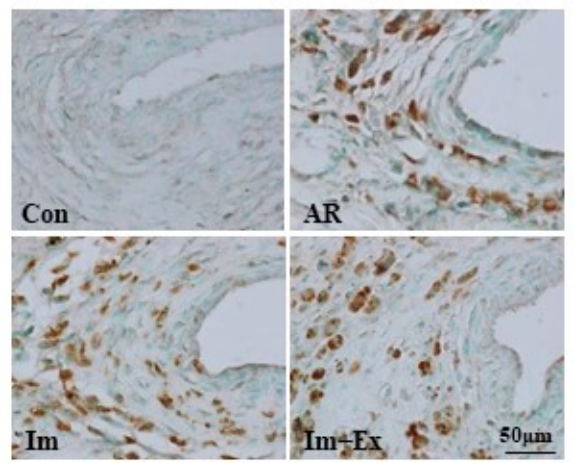

b) Von frey $15 \mathrm{~g}$

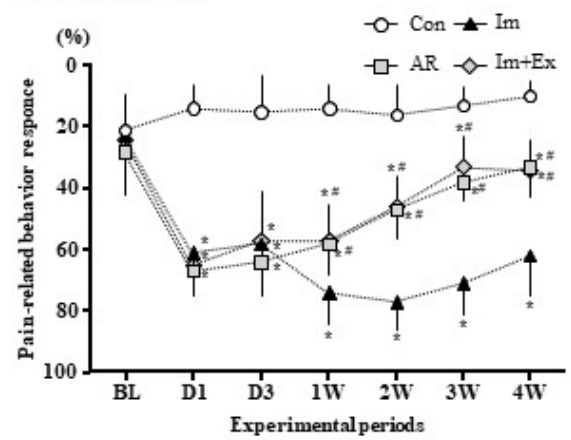

b) Changes of the number of CD68-positive cells

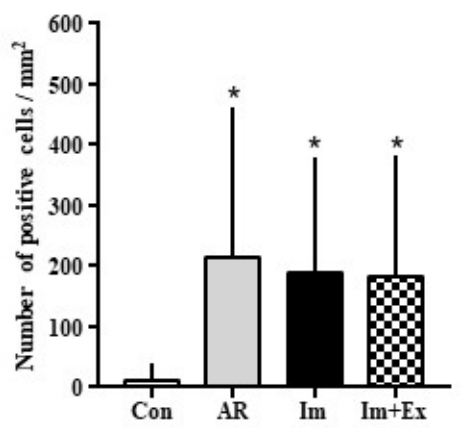

\section{a) Fluorescent immunostaining of CGRP}
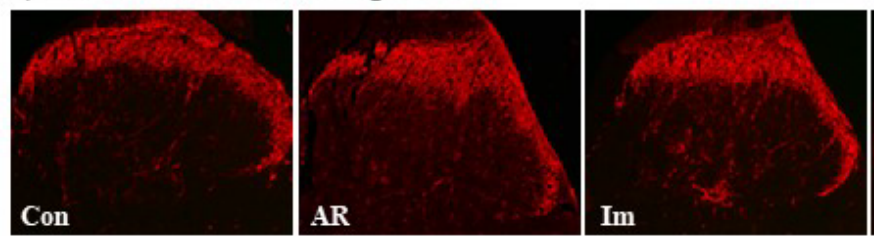

b) Changes of the percentage of fluorescence intensity of CGRP
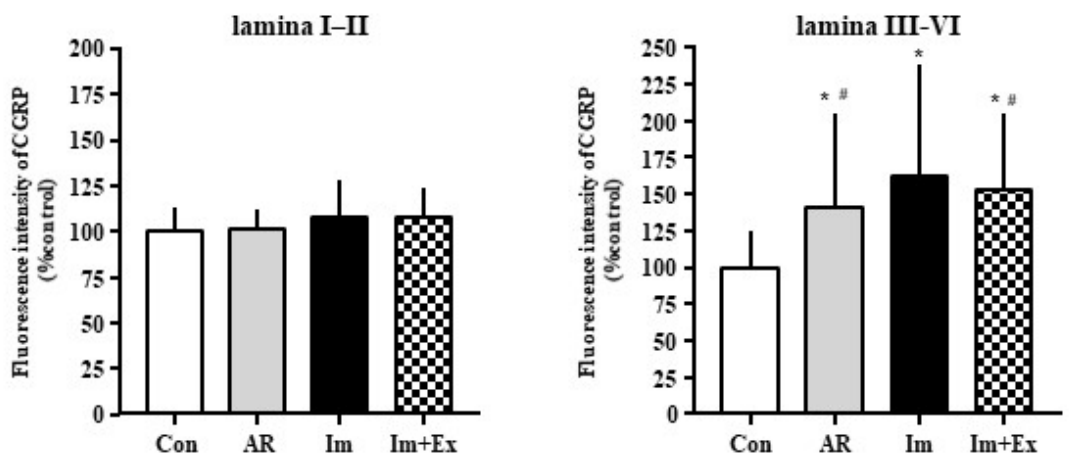

Fig. 3. Numbers of CD68-positive cells in the synovium of the knee joint. (a) Representative photographs of macrophage immunehistochemistry in the synovium. (b) Comparison of the number of CD68-positive cells per unit area $\left(1 \mathrm{~mm}^{2}\right)$. Data are mean $\pm \mathrm{SD}$. $*$ significantly different from the Con group $(p<0.05)$.

Fig. 2. Changes of the mechanical hyperalgesia of the hind paw in all groups. (a) $4 \mathrm{~g}$ von Frey filament (VFF) as a measurement of mechanical allodynia. (b) $15 \mathrm{~g}$ VFF as a measurement of mechanical hyperalgesia. Data are mean \pm SD. * significantly different from the Con group $(p<0.05)$; * significantly different from the Im group $(p<0.05)$.

Fig. 4. CGRP expression in the spinal dorsal horn at the L2-3. (a) Representative photographs of CGRP immunohistochemistry in the spinal dorsal horn at the L2-3. (b) Percentage of fluorescence intensity of CGRP expression in the superficial layer (lamina I-II) and deep layers (lamina III-VI) at L2-3. Data are mean $\pm \mathrm{SD}$. * significantly different from the Con group $(p<0.05) ; \quad+$ significantly different from the AR group $(p<0.05)$; \# significantly different from the Im group $(p<0.05)$. CGRP: calcitonin gene related peptide. 
In the spinal dorsal horns at L4-5, CGRP-positive neural fibers were clearly observed in the deep layers of the spinal dorsal horns in the AR and Im groups (Fig. 5a). In the superficial layer, there were no significant differences among the 4 groups. In the deep layer, the expression of CGRPs in the AR and Im groups significantly increased compared with that of the Con group. Im+Ex group significantly decreased when compared with the AR and Im groups and there were no significant differences with the Con group (Fig. 5b). a) Fluorescent immunostaining of CGRP

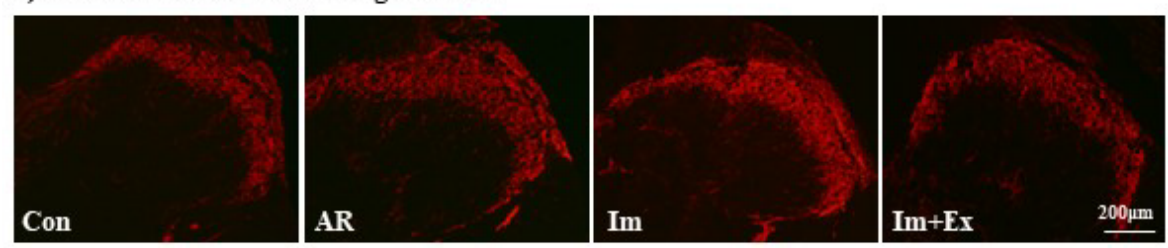

b) Changes of the percentage of fluorescence intensity of CGRP
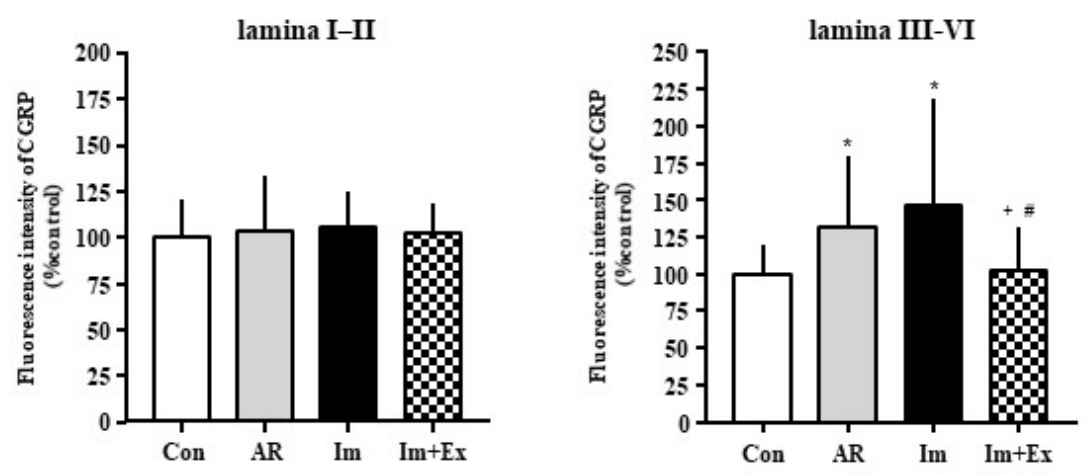

Fig. 5. CGRP expression in the spinal dorsal horn at the L4-5. (a) Representative photographs of CGRP immunohistochemistry in the spinal dorsal horn at the L4-5. (b) Percentage of fluorescence intensity of CGRP expression in the superficial layer (lamina I-II) and deep layers (lamina III-VI) at L4-5. Data are means $\pm \mathrm{SD}$. * significantly different from the Con group $(p<0.05) ; \quad+$ significantly different from the AR group $(p<0.05) ; \quad$ " significantly different from the Im group $(p<0.05)$. CGRP: calcitonin gene related peptide.

\section{Discussion}

This study used a rat experimental model of acute arthritis to simulate a clinical setting that required immobilization due to a marked inflammation of the affected area. Immobilization began on the same day as the injection of the inflammatory drug and continued for 4 weeks. On the $1^{\text {st }}$ week after immobilization, the swelling of the Im group significantly decreased compared to that of the AR group, and it returned to the same level as that of the Con group by the end of the experiment. These results suggest that immobilization after inflammation of the affected area may contribute to the recovery of swelling. Regarding this effect, we speculate that compression by the cast might reduce the swelling. However, the PPT of the Im group was significantly lower than that of the AR group during the experiment. Similarly, the PWR using $4 \mathrm{~g}$ and $15 \mathrm{~g}$ VFFs of the Im group was significantly higher than that of the AR group during the experiment. These results indicated that pain in the affected area and in distant areas was exacerbated by immobilization. Nakabayashi et al. performed a similar immobilization study, and their results also showed that immobilization caused delayed recovery from pain in the affected and distant areas
(Nakabayashi et al. 2016). In addition, the current study measured the sensitivity of the hind paws using $4 \mathrm{~g}$ VFFs for mechanical allodynia and $15 \mathrm{~g}$ VFFs for mechanical hyperalgesia. Therefore, the results of this study suggest that immobilization after arthritis may cause mechanical allodynia and hyperalgesia in distant areas.

The number of CD68-positive cells in the synovium in the Im group significantly increased when compared with that of the Con group. However, there were no significant differences in the numbers of CD68-positive cells between the Im and AR groups. Ishikawa et al. (2019) using an arthritis model with immobilization, reported that the numbers of CD68-positive cells in the synovium from arthritis did not change with immobilization. Therefore, the results of this study suggest that immobilization may not affect histological inflammation of the affected area. In addition, swelling was reduced in the Im group despite the remaining histological inflammation of the affected area. The remaining histological inflammation did not affect the degree of swelling. Even in the $4^{\text {th }}$ week postinjection, the Im group had persistent pain in the affected area, and we hypothesized that residual histological inflammation might be related to the pain in the affected area. Conversely, according to the results of the CGRP in 
L2-3 spinal dorsal horns, there were no significant differences in the expression of CGRP between the Im and AR groups in the superficial layer. Nakabayashi et al. (2016) reported that the expression of CGRP at L2-3 in the superficial layer did not increase after 8 weeks of immobilization after inducing arthritis in the rat knee joint. Although the immobilization periods were different, their report supported our results. In the deep layers, the expression of CGRPs in the Im group was significantly higher than that in the AR group, which also corresponded to the PPT results. In addition, according to the results of the CGRP in L4-5 spinal dorsal horns, the expression of CGRPs in the deep layers of the Im group was significantly increased compared with that of the Con group. These results suggest that the immobilization of the affected area may enhance the central sensitization in the spinel dorsal horn and increase primary and secondary hyperalgesia.

Next, this study examined the effects of wheelrunning using the upper limbs following immobilization after inducing arthritis in the knees of rats. On the $2^{\text {nd }}$ week post-injection, the PPT in the $\mathrm{Im}+\mathrm{Ex}$ group increased significantly compared with that in the Im group, and was higher than that in the Im group until the end of the experiment. This result suggested that EIH was caused by wheel-running exercises using upper limbs, which resulted in early relief of pain in the affected area. The PWR using $4 \mathrm{~g}$ VFFs in the Im+Ex group was significantly lower than that of the Im group from the $2^{\text {nd }}$ week post-injection. The PWR using $15 \mathrm{~g}$ VFFs in the Im+Ex group was significantly lower than that of the Im group from the $1^{\text {st }}$ week post-injection. These results suggest that EIH was caused by wheel-running exercises using upper limbs, which resulted in early relief of mechanical allodynia and hyperalgesia in the distant area. This study simulates a clinical setting that requires rest (immobilization) in the affected area and is the first report that voluntary movement by the upper extremities improves hyperalgesia in distant affected areas.

The number of CD68-positive cells in the synovium of the Im+Ex group was significantly increased compared with that of the Con group. However, there were no significant differences in the numbers of CD68-positive cells among the Im+Ex, Im, and AR groups. These results suggest that the effect of EIH by wheel-running exercises of the upper limbs did not promote recovery of histological inflammation in the affected area. However, pain in both the affected and distant areas was reduced by the wheel-running exercises using the upper limbs. Therefore, this may be influenced by central rather than peripheral mechanisms. Actually, Kami et al. (2017) also reported that EIH was a phenomenon which was induced through multiple cellular and molecular events produced in the central system following exercise. In the superficial layers of spinal dorsal horns, there were no differences in the expression of CGRPs on L2-3 and L4-5 between the Im+Ex and Im groups. In the deep layers of the spinal dorsal horns; however, the expression of CGRP in the Im+Ex group was significantly lower than that in the Im group. These results suggest that wheel running exercises of the upper limbs reduce central nerve sensitization of spinal dorsal horns in the affected and distant areas. Therefore, we presume that these phenomena affected pain relief in the affected and distant areas. In addition, all of these results indicated that exercise with the upper limbs may reduce primary and secondary hyperalgesia without affecting the inflammatory response in the affected areas.

Currently, the mechanisms responsible for EIH remain obscure; however, the endogenous opioid system has received significant attention. An experimental study using animals revealed that exercise can increase the $\beta$-endorphins in the RVM and PAG, and these phenomena are involved in the mechanism of EIH (Stagg et al. 2011). Therefore, previous study suggested that EIH might be induced by the upregulation of endogenous opioids in the brainstem. On the other hand, the non-opioid systems also have received significant attention. One of non-opioid systems is the endocannabinoid system (Dietrich and McDaniel 2004), a previous study showed that the endocannabinoid mechanism was involved in $\mathrm{EIH}$ following isometric exercise (Koltyn et al. 2014). However, it is still unknown whether EIH generated by various exercise protocols has its own characteristic mechanism, and further EIH research is necessary.

In summary, immobilization of the affected area for a period after the injury is essential; thus, in this case, the prevention of chronic pain by exercise using nonimmobilized upper limbs should be considered. According to the results of this study, upper limb exercise can decrease pain in the affected area, reduce hyperalgesia in distant areas, and suppress the central sensitization in the spinal dorsal horn by increasing the pain threshold and by triggering EIH. Therefore, exercise using non-immobilized limbs is a useful treatment strategy in the acute phase of tissue injury and a strategy to prevent the occurrence of chronic pain. However, this study has some limitations. First, the results are limited to 
an animal model and cannot be generalized to a human model. Second, in order to clarify whether the exercise improved inflammation- and/or immobilization-induced hyperalgesia, it was necessary to add the group which loads exercise after arthritis induction and to analyze. Third, this research evaluated the number of macrophages as a biomarker for the inflammation response in the synovitis. But this alone could not sufficient to evaluate histological inflammation, and the changes of inflammatory cytokines such as IL- $1 \beta$ and TNF- $\alpha$ should be evaluated in the future. Similarly, this study examined the expression of CGRPs in the spinal dorsal horns; however, there are numerous factors involved in the central sensitization such as the activation of the glial cells and expression of other neurotransmitters such as substance P, nitric oxide, and glutamate. Fourth, while previous studies have reported that various brain mechanisms are involved in the mechanism of EIH (Chuganji et al. 2015, Stagg et al. 2011), this was not addressed in the present study. Therefore, it is necessary to evaluate these parameters in the future.

\section{Conflict of Interest}

There is no conflict of interest.

\section{References}

CHUGANJI S, NAKANO J, SEKINO Y, HAMAUE Y, SAKAMOTO J, OKITA M: Hyperalgesia in an immobilized rat hindlimb: effect of treadmill exercise using non-immobilized limbs. Neurosci Lett 584: 66-70, 2015. https://doi.org/10.1016/j.neulet.2014.09.054

DIETRICH A, MCDANIEL WF: Endocannabinoids and exercise. $\mathrm{Br} \mathrm{J}$ Sports Med 38: 536-541, 2004. https://doi.org/10.1136/bjsm.2004.011718

ISHIKAWA K, KAJIWARA Y, SAKAMOTO J, SASAKI R, GOTO K, HONDA Y, KATAOKA H, OKITA M: Low-intensity muscle contraction exercise following the onset of arthritis improves hyperalgesia via reduction of joint inflammation and central sensitization in the spinal cord in a rat model. Neurosci Lett 706: 18-23, 2019. https://doi.org/10.1016/j.neulet.2019.04.031

KAMI K, TAJIMA F, SENBA E: Exercise-induced hypoalgesia: potential mechanisms in animal models of neuropathic pain. Anat Sci Int 92: 79-90, 2017. https://doi.org/10.1007/s12565-016-0360-z

KAMI K, TAGUCHI S, TAJIMA F, SENBA E: Mechanisms and effects of forced and voluntary exercises on exerciseinduced hypoalgesia in neuropathic pain model mice. Pain Res 30: 216-229, 2015. https://doi.org/10.11154/pain.30.216

KAWAI S, TAKAGI Y, KANEKO S, KUROSAWA T: Effect of three types of mixed anesthetic agents alternate to ketamine in mice. Exp Anim 60: 481-487, 2011. https://doi.org/10.1538/expanim.60.481

KOLTYN KF, BRELLENTHIN AG, COOK DB, SEHGAL N, HILLARD C: Mechanisms of exercise-induced hypoalgesia. J Pain 15: 1294-1304, 2014. https://doi.org/10.1016/j.jpain.2014.09.006

MOLANDER C, XU Q, RIVERO-MELIAN C, GRANT G: Cytoarchitectonic organization of the spinal cord in the rat: II. The cervical and upper thoracic cord. J Comp Neurol 289: 375-385, 1989. https://doi.org/10.1002/cne.902890303

NAKABAYASHI K, SAKAMOTO J, KATAOKA H, KONDO Y, HAMAUE Y, HONDA Y, NAKANO J, OKITA M: Effect of continuous passive motion initiated after the onset of arthritis on inflammation and secondary hyperalgesia in rats. Physiol Res 65: 683-691, 2016. https://doi.org/10.33549/physiolres.933214

NAKANO J, SEKINO Y, HAMAUE Y, SAKAMOTO J, YOSHIMURA T, ORIGUCHI T, OKITA M: Changes in hind paw epidermal thickness, peripheral nerve distribution and mechanical sensitivity after immobilization in rats. Physiol Res 61: 643-647, 2012. https://doi.org/10.33549/physiolres.932362

NAUGLE KM, FILLINGIM RB, RILEY JL: A meta-analytic review of the hypoalgesic effects of exercise. J Pain 13: 1139-1150, 2012. https://doi.org/10.1016/j.jpain.2012.09.006

NEUGEBAUER V, HAN JS, ADWANIKAR H, FU Y, JI G: Techniques for assessing knee joint pain in arthritis. Mol Pain 3: 8, 2007. https://doi.org/10.1186/1744-8069-3-8

O'CONNOR SR, TULLY MA, RYAN B, BLEAKLEY CM, BAXTER GD, BRADLEY JM, MCDONOUGH SM: Walking exercise for chronic musculoskeletal pain: systematic review and meta-analysis. Arch Phys Med Rehabil 96: 724-734.e3, 2015. https://doi.org/10.1016/j.apmr.2014.12.003 
PELESHOK JC, RIBEIRO-DA-SILVA A: Delayed reinnervation by nonpeptidergic nociceptive afferents of the glabrous skin of the rat hindpaw in a neuropathic pain model. J Comp Neurol 519: 49-63, 2011. https://doi.org/10.1002/cne.22500

RADHAKRISHNAN R, MOORE SA, SLUKA KA: Unilateral carrageenan injection into muscle or joint induces chronic bilateral hyperalgesia in rats. Pain 104: 567-577, 2003. https://doi.org/10.1016/s0304-3959(03)00114-3

STAGG NJ, MATA HP, IBRAHIM MM, HENRIKSEN EJ, PORRECA F, VANDERAH TW, MALAN TP JR: Regular exercise reverses sensory hypersensitivity in a rat neuropathic pain model: role of endogenous opioids. Anesthesiology 114: 940-948, 2011. https://doi.org/10.1097/aln.0b013e318210f880

TAJERIAN M, CLARK JD: Nonpharmacological interventions in targeting pain-related brain plasticity. Neural Plast 2017: 2038573, 2017. https://doi.org/10.1155/2017/2038573

VAEGTER HB, HANDBERG G, GRAVEN-NIELSEN T: Similarities between exercise-induced hypoalgesia and conditioned pain modulation in humans. Pain 155: 158-167, 2014. https://doi.org/10.1016/j.pain.2013.09.023 